\title{
Probing the Material Loss and Optical Nonlinearity of Integrated Photonic Materials
}

\author{
Maodong Gao ${ }^{1, \dagger}$, Qi-Fan Yang ${ }^{1, \dagger}$, Qing-Xin $\mathrm{Ji}^{1}$, Lue Wu ${ }^{1}$, Junqiu Liu ${ }^{2}$, Guanhao Huang ${ }^{2}$, \\ Lin Chang ${ }^{3}$, Weiqiang Xie ${ }^{3}$, Boqiang Shen ${ }^{1}$, Heming Wang ${ }^{1}$, Zhiquan Yuan ${ }^{1}$, Su-Peng \\ $\mathbf{Y u}^{4}$, Scott Papp ${ }^{4}$, Tobias Kippenberg ${ }^{2}$, John Bowers ${ }^{3}$ and Kerry Vahala ${ }^{1, *}$ \\ ${ }^{1}$ T. J. Watson Laboratory of Applied Physics, California Institute of Technology, Pasadena, CA 91125, USA \\ ${ }^{2}$ Institute of Physics, Swiss Federal Institute of Technology Lausanne (EPFL), CH-1015 Lausanne, Switzerland \\ ${ }^{3}$ ECE Department, University of California Santa Barbara, Santa Barbara, CA 93106, USA \\ ${ }^{4}$ National Institute of Standards and Technology, Boulder, CO, USA \\ *vahala@caltech.edu
}

\begin{abstract}
Optical absorption and nonlinear index are important performance drivers in devices like microcombs and parametric oscillators. Here we use resonance-enhanced nonlinear spectroscopy to characterize absorption limits and nonlinear index for some integrated photonic materials. (c) 2021 The Author(s)
\end{abstract}

The threshold for Kerr-induced parametric oscillation in a microresonator is of critical importance to soliton microcomb operation. It determines the comb operating power and also figures prominently in other parameters such as the soliton existence pump-detuning range [1]. Threshold power scales approximately inverse-quadratically with the microresonator $Q$ factor and inversely with the nonlinear Kerr index [2]. It is therefore interesting to understand the impact of resonator material selection on these two parameters; and, in turn, to estimate a best-case threshold power/mode-volume. Because optical absorption and the Kerr effect each cause a change in refractive index (the former through the photothermal effect), cavity resonance frequency will shift versus coupled optical power through the sum contribution of these two mechanisms. Indeed, absorption-induced shifts caused by the photo-thermal effect in silica (which features a very weak Kerr effect) have been used to assess an upper bound (i.e., without scattering and waveguide loading) to $Q$ factor provided by absorption [3]. On the other hand, isolation of Kerr and photothermal frequency-shift contributions is possible by measuring the frequency response for transfer of pump power modulation onto a probe signal (each tuned to different cavity resonances) [4]. And, the ratio of these mechanisms inferred by this measurement also provides the absorptive-limited $\mathrm{Q}$ factor when combined with a known value of the Kerr coefficient [5]. Here, these two methods (sum and ratio) are combined so that both the Kerr nonlinear index and the absorption limit to $Q$ factor can be measured in several integrated photonic material systems.

The experimental setup for the sum measurement is shown in Fig. 1(a). This quasi static measurement is performed by slowly scanning the frequency of a laser across a cavity resonance from blue-to-red. Examples of the resulting transmission spectra scans, which feature a well known triangular profile, are presented in Fig. 1(b) for silica $\left(\mathrm{SiO}_{2}\right)$ [6], silicon-nitride $\left(\mathrm{Si}_{3} \mathrm{~N}_{4}\right)$ [5], tantala $\left(\mathrm{Ta}_{2} \mathrm{O}_{5}\right)$ [7], and aluminium gallium arsenide $\left(\mathrm{Al}_{0.2} \mathrm{Ga}_{0.8} \mathrm{As}\right)$ [8]

(a)

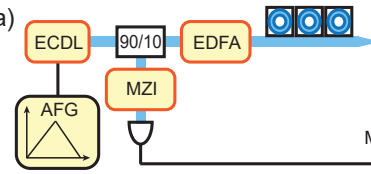

(b)

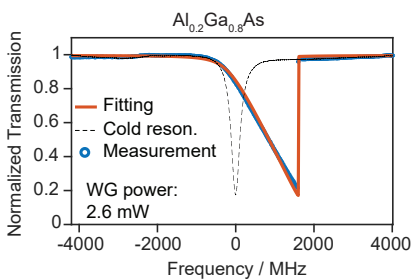

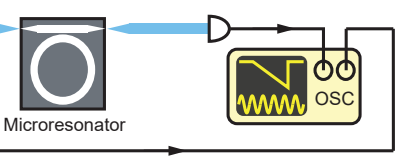

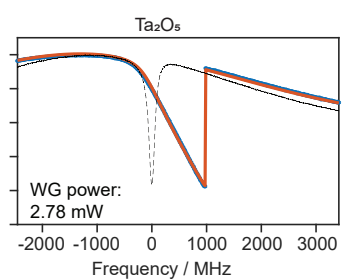

(c)
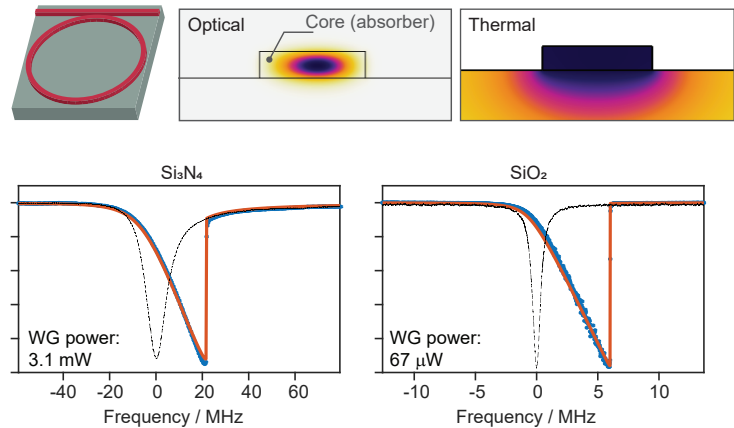

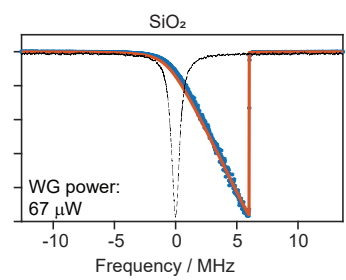

Fig. 1. (a) Experimental setup for the 'sum' measurement. AFG: arbitrary function generator. ECDL: external-cavity diode laser. EDFA: erbium-doped fiber amplifier. MZI: Mach-Zehnder interferometer. OSC: oscilloscope. (b) Quasi-static frequency broadening measurement performed on four resonators fabricated from the indicated materials. A theoretical fitting is shown as the dashed curve and accounts for reflection by the two coupling facets of the waveguide. Cold-cavity resonance (i.e., Kerr-effect only) is also shown for comparison. WG: waveguide. (c) Illustration showing a microresonator, its optical mode, and the temperature plot associated with photothermal heating (darker areas indicate higher temperature). 
(a)

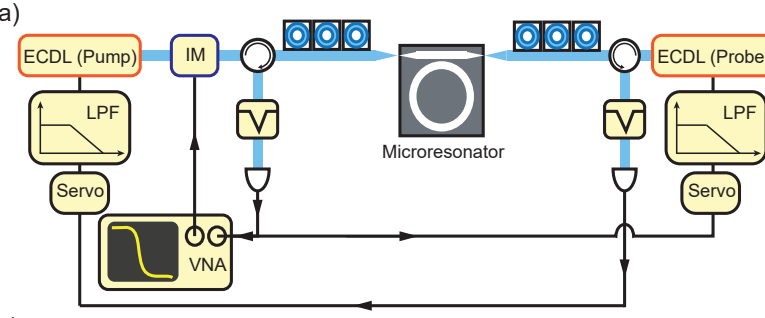

(c)

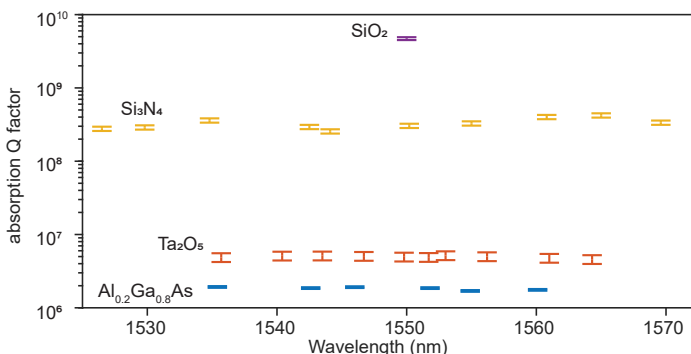

(b)

(d)
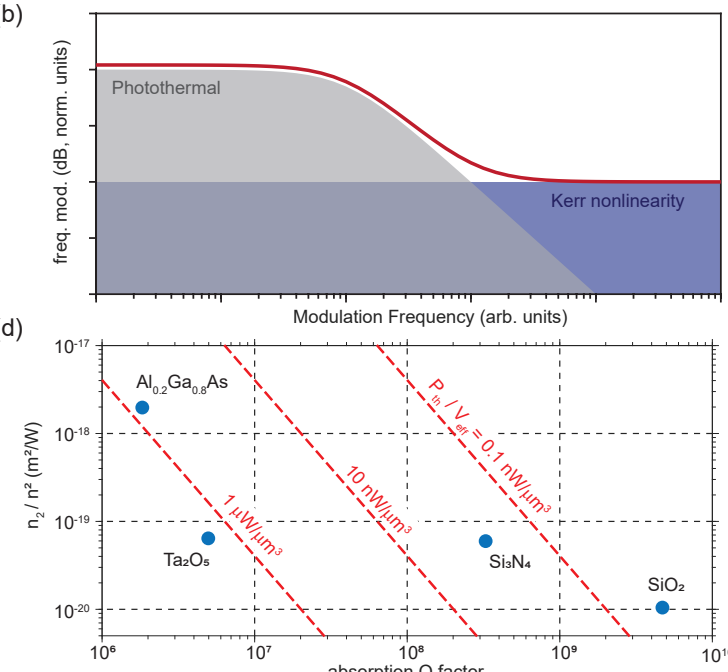

Fig. 2. (a) Experiment setup for the 'ratio' measurement. IM: intensity modulator. LPF: low pass filter. VNA: vector network analyzer. (b) Illustration of cavity frequency modulation response curve as a function of input-power modulation frequency showing photothermal and Kerr contributions. (c) Measured absorption $Q$ factors at different wavelengths in the C-band for the four materials (colors). Vertical error bars give measurement standard deviations. (d) Comparison of absorption $Q$ factors and normalized nonlinear coefficients $\left(n_{2} / n^{2}\right)$ for the four materials. Measured $n_{2}$ values are, in units of $10^{-20} \mathrm{~m}^{2} / \mathrm{W}: \mathrm{Al}_{0.2} \mathrm{Ga}_{0.8} \mathrm{As}, 1616 \pm 43 ; \mathrm{Ta}_{2} \mathrm{O}_{5}, 26.0 \pm 3.6$; and $\mathrm{Si}_{3} \mathrm{~N}_{4}, 21.1 \pm 1.4$. The $n_{2}$ of $\mathrm{SiO}_{2}$ was not measured here and a value of $2.2 \times 10^{-20} \mathrm{~m}^{2} / \mathrm{W}$ is used. Parametric oscillation threshold normalized by mode volume $\left(\mathrm{P}_{\mathrm{th}} / \mathrm{V}_{\text {eff }}\right)$ is indicated by the red dashed lines and assumes: $\lambda=1550 \mathrm{~nm}$, intrinsic $Q$ equals material absorption $Q$, and critical coupling condition.

high-Q microresonators. Waveguide power levels are provided in the legends of Fig. 1(b). This measurement and the ratio measurement must be supplemented by numerical analysis that incorporates thermal diffusion in combination with the optical mode profile (as indicated in Fig. 1(c)). The experimental setup for the ratio measurement is shown in Fig. 2(a). An illustration of the corresponding frequency response is provided in Fig. 2(b). It shows how at low (high) modulation rates the photothermal (Kerr) effect is dominant.

Combining results from the sum (via spectral triangle) and ratio (via modulation response) measurements, the photothermal and Kerr coefficients can be obtained individually. Both measurements are carried out on multiple modes across the optical C-band, and the measured absorption-limited $Q$ values for each material are summarized in Fig. 2(c). As an aside, the silica measurement required a narrow-linewidth fiber laser on account of the ultrahigh- $Q$ of the silica microresonators, and this restricted the measurement tuning range as indicated. For the other materials tested, the absorption $Q$ factors do not show significant wavelength dependencies over the C-band. The measured $n_{2}$ values (normalized by $n^{2}$ in accordance with the dependence in the parametric oscillation threshold formula), and the absorption Q are plotted in Fig. 2(d) (the silica $n_{2}$ is from the literature). Parametric oscillation threshold per unit mode volume is indicated in contours (Fig. 2(d)).

The $\mathrm{Q}$ measurements presented here should be viewed as state-of-the-art (current performance) values for these wafer based materials and not fundamental limits. $\mathrm{Ta}_{2} \mathrm{O}_{5}$ and $\mathrm{Al}_{0.2} \mathrm{Ga}_{0.8} \mathrm{As}$, for example, are emerging material platforms for high-Q resonators. And we anticipate that the methods presented here will enable their rapid improvement and optimization.

We acknowledge support from AFOSR (FA9550-18-1-0353) and the Caltech Kavli Nanoscience Institute.

\section{References}

1. T. Herr, V. Brasch et. al. Nature Photonics 8, 145 (2014)

2. T. J. Kippenberg, S. M. Spillane, and K. J. Vahala Phys. Rev. Lett. 93, 083904 (2004)

3. H. Rokhsari, S. M. Spillane and K. J. Vahala, Appl. Phys. Lett., 85, 3029 (2004)

4. H. Rokhsari and K. Vahala, Optics Letters, 30, 427-429 (2005)

5. J. Liu et. al. arXiv: 2005.13949 (2020).

6. L. Wu et. al Optics Letters, 45, 5129-5131, (2020)

7. H. Jung et. al. arXiv:2007.12958 (2020)

8. L. Chang et. al. Nat. Commun. 11, 1331 (2020). 